\title{
BMJ Open Quality Improving the use of the 'COUGH' bundle in Surgical High Dependency Unit, Ninewells Hospital, Dundee
}

\author{
Victoria Livie, Jennifer Livie, Sharon Hilton-Christie
}

To cite: Livie V, Livie J, HiltonChristie S. Improving the use of the 'COUGH' bundle in Surgical High Dependency Unit, Ninewells Hospital, Dundee. BMJ Open Quality 2020;9:e000851. doi:10.1136/ bmjoq-2019-000851

Received 8 October 2019 Revised 12 April 2020 Accepted 18 April 2020

\section{Check for updates}

(c) Author(s) (or their employer(s)) 2020. Re-use permitted under CC BY-NC. No commercial re-use. See rights and permissions. Published by BMJ.

Surgical High Dependency Unit, Ninewells Hospital, NHS Tayside, Dundee, UK

Correspondence to

Dr Victoria Livie;

v.livie1@nhs.net

\section{ABSTRACT}

Developing respiratory complications postoperatively is one of the major determinants of longer hospital stay, morbidity, mortality and increased healthcare costs. The incidence of postoperative respiratory complications varies from $1 \%$ to $23 \%$. Given that postoperative respiratory complications are relatively common and costly, there have been various studies which look at ways to reduce the risk of these occurring. One such protocol is the ICOUGH bundle which stands for Incentive spirometry, Coughing and deep breathing, Oral care, patient Understanding, Getting out of bed and Head of bed elevation. This has been adapted locally to the Coughing and deep breathing, Oral care, patient Understanding, Getting out of bed and Head of bed elevation (COUGH) bundle which consists of these components excluding incentive spirometry. Within our surgical high dependency unit (HDU), the COUGH bundle should be implemented in patients who have a moderate or high risk of developing postoperative respiratory complications with an Assess Respiratory Risk in Surgical Patients in Catalonia (ARISCAT) score of 26 or above. Studies have shown that the ICOUGH bundle has reduced rates of pneumonia and unplanned intubation in general surgical and vascular patients. Baseline data taken from surgical HDU showed that the COUGH bundle was not well implemented. One out of eight patients who had an ARISCAT score greater than 26 had the COUGH bundle implemented on admission to the unit. Three out of eight patients had the ARISCAT score documented in their admission medical review. One patient who should have received the bundle, but did not, developed a hospital acquired pneumonia postoperatively. To address this issue, we aimed to increase awareness surrounding the COUGH bundle and to increase the number of patients who had the COUGH bundle started on admission. This quality improvement project had four cycles (plan, do, study, act) and after these, $100 \%$ of patients who had an ARISCAT score of 26 or more had the COUGH bundle implemented.

\section{PROBLEM}

Developing postoperative respiratory complications is one of the major determinants of longer hospital stay, morbidity, mortality and increased healthcare costs. ${ }^{1}$ Respiratory complications are common postoperatively with an incidence of between $1 \%$ and $23 \% .^{2}$ Mortality is also increased both in the short and long terms. Approximately 14\%-30\% of patients who have a postoperative respiratory complication will die within 30 days of major surgery compared with $0.2 \%-3 \%$ without. $^{2}$ Studies have also shown that postoperative pulmonary complications are the most costly and one study has found that treatment of these is $50 \%$ greater than costs for treating postoperative cardiac complications. ${ }^{3}$ The most important postoperative respiratory complications are reintubation, acute respiratory failure, pulmonary oedema, pneumonia and atelectasis. ${ }^{4}$

The Incentive spirometry, Coughing and deep breathing, Oral care, patient Understanding, Getting out of bed and Head of bed elevation (ICOUGH) bundle was developed to reduce respiratory complications postoperatively and studies have shown that the bundle has reduced the incidence of postoperative pneumonia and unplanned intubations. ${ }^{5}$ This was adapted locally to the Coughing and deep breathing, Oral care, patient Understanding, Getting out of bed and Head of bed elevation (COUGH) bundle. Within our surgical high dependency unit (HDU), it is used in patients who have an Assess Respiratory Risk in Surgical Patients in Catalonia (ARISCAT) score of 26 or above. ${ }^{6}$

Ninewells Hospital is a large teaching hospital located within Dundee, Scotland. The 10-bedded surgical HDU receives patients from a wide variety of surgical specialties including general surgery, vascular surgery, urology, orthopaedics, gynaecology and plastic surgery. On a day-to-day basis, the team is led by a consultant anaesthetist; however, many different healthcare professionals work within the unit including surgeons, junior doctors, nurses, physiotherapists and dieticians. Within the surgical HDU department, the COUGH bundle was not well implemented. Baseline data revealed that only one out of eight patients had the COUGH bundle started on admission to surgical HDU. One patient who should have had the COUGH bundle implemented, but did not receive it, developed hospital acquired 
pneumonia postoperatively. Respiratory infection can be defined by the European Perioperative Clinical Outcome definition as antibiotics for suspected infection with one or more of the following: new or changed sputum, new or changed lung opacities, fever, white cell count greater than $12 \times 10^{9} / \mathrm{L}^{2}$ A study performed in the UK looked at 268 consecutive major elective abdominal operations in 6 hospitals. Results showed that postoperative pulmonary complications were reported in $11.9 \%$ of cases at 7 days with pulmonary infection being the most common at $9 \%{ }^{7}$ Within this study, postoperative pulmonary complications increased the median length of hospital stay by 7 days. ${ }^{7}$

Given that research shows that postoperative respiratory complications are common, costly and increases mortality, the aim of the project was to increase the use of the COUGH bundle so that at least $80 \%$ of postoperative patients who had an ARISCAT score of 26 or more were started on the bundle on admission by 4 December 2018 .

\section{BACKGROUND}

Postoperative respiratory complications are common with the incidence varying between $1 \%$ and $23 \%{ }^{2}$ Various respiratory complications exist including atelectasis, pneumonia, respiratory failure, pleural effusion, pneumothorax, aspiration pneumonitis, acute respiratory distress syndrome (ARDS) and pulmonary embolism. ${ }^{2}$ There have been many studies which look at the incidence of postoperative pulmonary complications. A study performed looking at 1673 patients who underwent non-cardiac surgery had a total of $163(9.7 \%)$ patients developing postoperative pulmonary complication. ${ }^{8}$ Out of these patients, respiratory infection was seen in $7.8 \%$ and respiratory failure developed in $1.8 \%$ of patients. ${ }^{8}$ Another study which involved 1170 patients who underwent non-cardiac surgery had a postoperative pulmonary complication rate of $5 \% .{ }^{9}$ Out of these patients, pulmonary infection was the most common followed by atelectasis, bronchospasm and pulmonary embolism. ${ }^{9}$

Developing postoperative respiratory complications is one of the major determinants of longer hospital stay, morbidity, mortality and increased healthcare costs. ${ }^{1}$ Mortality is increased both in the short and long terms in patients who develop a postoperative respiratory complication. A study involving 2464 patients undergoing surgical procedures showed that 30-day mortality was higher in patients with a postoperative pulmonary complication at $19.5 \%$ than those without at $0.5 \% .{ }^{10}$ At 90 days, the mortality was also increased in patients who had a respiratory complication at $24.4 \%$ compared with $1.2 \%$ who did not have a postoperative pulmonary complication. ${ }^{10} \mathrm{~A}$ study looking at postoperative pulmonary complications found that early mortality rate was the highest in patients with ARDS and reintubation with postoperative mechanical ventilation but was also associated with pneumonia, pleural effusion and atelectasis. ${ }^{11}$ Studies have shown that postoperative pulmonary complications are the most costly and one study has found that treatment of these is
$50 \%$ greater than costs for treating postoperative cardiac complications. ${ }^{3}$ Healthcare costs are also increased as the average length of stay for patients developing postoperative respiratory complications increases up to a further 8 days. ${ }^{12}$ A study involving 450 patients found that length of hospital stay was increased in those patients who developed postoperative pulmonary complications (7.48 days vs 3.97 days). ${ }^{13}$

There are many preoperative risk factors for the development of postoperative respiratory complications. These can be largely divided into patient-related and procedure-related risk factors. Examples of patientrelated risk factors include age over 65 years, smoking, obesity, comorbidities such as chronic obstructive pulmonary disease, asthma and obstructive sleep apnoea. ${ }^{14}$ Procedure-related risk factors include duration of surgery, type of anaesthesia, type of surgery and site of surgery. ${ }^{14}$ The ARISCAT score uses a seven-factor scoring system to assess the risk of a postoperative respiratory complication. Studies have shown that ARISCAT score is a good predictor of risk for developing postoperative pulmonary complications in patients undergoing major surgery. ${ }^{15}$ The seven factors include age, preoperative oxygen saturations, respiratory infection in the last month, preoperative anaemia, surgical incision (peripheral, upper abdominal, intrathoracic), duration of surgery and whether the surgery was an emergency. ${ }^{6}$ Scores are calculated and if the score is 26 or more then patients have a moderate or high risk of developing a postoperative respiratory complication.

Intraoperatively, there are many pathophysiological changes that occur under anaesthesia and following surgery which contribute to developing respiratory complications. ${ }^{1}$ The change in body position from upright to supine reduces the rest lung volume by around $1 \mathrm{~L}$ and most general anaesthetic agents will cause a further reduction of functional residual capacity. ${ }^{1}$ Furthermore, patients who undergo abdominal surgery will have a $40 \%$ reduction in functional residual, total lung capacity and forced expired volume in the first second for at least 7 days. ${ }^{1}$ Atelectasis that occurs in more than $75 \%$ of patients undergoing a general anaesthetic involving a neuromuscular blocking drug can lead to pneumonia and acute respiratory failure. ${ }^{12}$

There has been a plethora of research looking into ways of how to reduce postoperative pulmonary complications. Preoperative strategies include optimising patients' respiratory disease prior to an operation and early smoking cessation. ${ }^{16}$ Prolonged abstinence from smoking significantly decreases the risk of postoperative respiratory complications. Six months of abstinence restores antimicrobial and inflammatory alveolar macrophage function. ${ }^{17}$ Using alternative anaesthetic (such as regional or neuroaxial rather than general anaesthetic) and modified surgical techniques (minimally invasive surgical procedures instead of open surgeries) and limiting the duration of surgery should be considered in high-risk patients. $^{9} 14$ 
Postoperative strategies include early mobilisation, effective analgesia, lung expansion techniques and chest physiotherapy. ${ }^{16}$ Inadequate analgesia prevents early mobilisation and deep breathing; thus, it is important to choose an analgesic method which can improve pain and pulmonary function. ${ }^{18}$ A study looking at epidural analgesia found that in patients undergoing abdominal or thoracic surgery, epidural analgesia decreased the risk of postoperative pneumonia. ${ }^{19}$ Postoperative manoeuvres to increase mean lung volumes are of benefit in preventing pulmonary complications. ${ }^{20}$ Techniques include chest physiotherapy, deep breathing exercises, cough, postural draining, percussion and vibration, suctioning and ambulation, incentive spirometry and continuous positive airway pressure (CPAP). ${ }^{16}$ Despite incentive spirometry being commonly used postoperatively, a systematic review of 30 studies shows that there was no evidence to support the use of incentive spirometry in the management of surgical patients. ${ }^{21}$ CPAP in patients who develop hypoxaemia postoperatively after major abdominal surgery may reduce the need for intubation and incidence of pneumonia. ${ }^{16}$ A trial looking at the efficacy of a single preoperative physiotherapy session showed that the incidence of postoperative pulmonary complications, including hospital acquired pneumonia, was halved. ${ }^{22}$ The preoperative physiotherapy session lasted 30 minutes and involved breathing exercise training as well as education focused on respiratory complications and their prevention. ${ }^{22}$

There has been research looking at bundles which help to reduce postoperative pulmonary complications. A study looking at patients undergoing general and vascular surgery found that the ICOUGH bundle reduced the incidence of postoperative pneumonia from $2.6 \%$ to $1.6 \%$ and the incidence of unplanned intubations fell from $2 \%$ to $1.2 \%$ after the bundle was implemented. ${ }^{5}$ Another bundle called Enhanced Recovery After Surgery+ has also been developed and this is a model based on combining elements of enhanced recovery with specific measures aimed at reducing pulmonary complications. ${ }^{23}$ Data was collected from 801 patients who scored 26 or more on the ARISCAT score and there was a reduction in postoperative pulmonary complications from $19.3 \%$ to $8.7 \%$ oneyear after the bundle was implemented..$^{23}$ There is other research being carried out currently in order to reduce postoperative pulmonary complications. One example is the PRIME-AIR study which is a trial focusing on an anaesthesia-centred bundle to reduce postoperative pulmonary complications.

\section{MEASUREMENT}

The primary outcome measure was the percentage of postoperative patients who had the COUGH bundle implemented in patients with an ARISCAT score of more than 26 on admission to surgical HDU. For the COUGH bundle to be fully implemented, it was expected that three components were done. The first component of the COUGH bundle was that the ARISCAT score was documented in the surgical HDU admission sheet. The second component of the COUGH bundle was to ensure that mouthwash was prescribed two times a day on the drug Kardex. The third component of the COUGH bundle was an observation chart completed daily by nursing staff or physiotherapists. This observation chart documented when the patient was encouraged to perform coughing and deep breathing exercises and when the patient was mobilised into a chair.

Due to only 10 beds being in the unit and a variable number of beds being filled at any one time, baseline data was collected over 1 week. Baseline data revealed that only one patient out of eight $(12.5 \%)$ had the COUGH bundle fully implemented. A further two patients had the ARISCAT score and chlorhexidine mouthwash prescribed but the observation chart was not completed. One patient who should have received the COUGH bundle, but did not, developed a hospital acquired pneumonia.

\section{DESIGN}

The project team consisted of a consultant anaesthetist who worked shifts in the surgical HDU as well as two junior doctors who spent time in the unit during their surgical block. It was important to engage with members of the unit to ensure that the project was successful as well as sustainable in the future. It was the role of the junior doctor to identify patients who were suitable for the COUGH bundle by calculating their ARISCAT score when clerking the patient on admission. Following this, an observation chart should be given to nursing staff or physiotherapists and chlorhexidine mouthwash should be prescribed on the drug Kardex.

The project consisted of four PDSA (plan, do, study, act) cycles. It was important to inform all members of the team who worked in the unit regarding the project; therefore, an email was sent out detailing their roles to help ensure the COUGH bundle was being used. Other interventions included creation of posters and a sticker placed in the surgical admission clerking document. It was felt the sticker in the clerking document was the most sustainable intervention as the ward clerk played a key role in making sure it was applied to every admission sheet. While junior doctors rotate throughout the unit on a 4 monthly basis, the ward clerk is one of the long standing members of staff who can ensure the project runs on.

\section{STRATEGY}

The aim of the project was to increase the use of the COUGH bundle so that at least $80 \%$ of patients who had an ARISCAT score of 26 or more were started on the bundle on admission to the surgical HDU department by 4 December 2018. The project started on the 16 September 2018. Collection of data for each PDSA cycle was over 1 week. In total, there were four PDSA cycles which took place between 16 September and 4 December. These are as follows: 


\section{PDSA cycle 1}

The first PDSA cycle involved informing members of the multidisciplinary team who worked in surgical HDU regarding this quality improvement project. The email was sent out to all general surgery junior doctors, anaesthetists and the senior charge nurse who distributed this further to nursing staff and physiotherapists within the unit. Within the email itself, each discipline's role in the project was highlighted thus ensuring members of staff knew what was expected of them. The percentage of patients who had the COUGH bundle implemented increased from $12.5 \%$ at baseline data to $14 \%$ at the end of cycle 1. In order to improve this, a further cycle was developed to focus on improving the documentation of the ARISCAT score. This was felt to be important as this identifies those patients who needed to have the COUGH bundle implemented.

\section{PDSA cycle 2}

The second PDSA cycle involved creating two postersone detailing the seven components which make up the ARISCAT score and another poster detailing the steps which the junior doctors need to undertake if the score is 26 or above. These included prescribing the chlorhexidine mouthwash two times daily on the Kardex and providing nursing staff and physiotherapists with the observation chart to complete. Both posters were hung on the wall beside the desktop computer which junior doctors work at. The percentage of patients who had the COUGH bundle implemented fell from $14 \%$ to $0 \%$. The observation chart was not filled in for any patients during this PDSA cycle; therefore, more improvement was needed to be done on this aspect. Prior to PDSA cycle 3 , discussions took place with the consultant anaesthetist and nurse in charge regarding further improvement in the project. It was decided at this point that a section for quality improvement project work would be made visible on the wall beside the doctor's computer.

\section{PDSA cycle 3}

The third PDSA cycle involved moving the observation charts from a filing cabinet to the wall beside the doctor's computer. This wall was dedicated for quality improvement projects going on in the unit. This wall was also in a different part of the ward to the filing cabinet and it was hypothesised that by moving the chart to a more visible and accessible space then it would be more likely to be completed. The percentage of patients who had the COUGH bundle implemented remained at $0 \%$ and zero observation charts were filled out. In order to improve on these data, it was felt a sticker in the admission document which junior doctors filled out would ensure that more responsibility was taken to ensure the various components of the COUGH bundle were being carried out.

\section{PDSA cycle 4}

The fourth PDSA cycle involved creating a sticker which was placed in the medical admission clerking document. This involved getting the junior doctor to document the ARISCAT score and circle Y/N (yes/no) if chlorhexidine mouthwash was prescribed on the Kardex and whether the observation chart had been given to nursing staff/ physiotherapists. It was hypothesised that this sticker would be a simple but effective reminder for medical staff to implement the COUGH bundle in the appropriate patients. There was an increase so that $100 \%$ of patients had the COUGH bundle implemented.

\section{RESULTS}

The primary outcome measure was the percentage of postoperative patients who had the COUGH bundle implemented in patients with an ARISCAT score of 26 or more on admission to surgical HDU. After four PDSA cycles, the percentage of patients who had the COUGH bundle implemented increased from $12.5 \%$ to $100 \%$ (see figure 1). The sticker in the surgical admission document

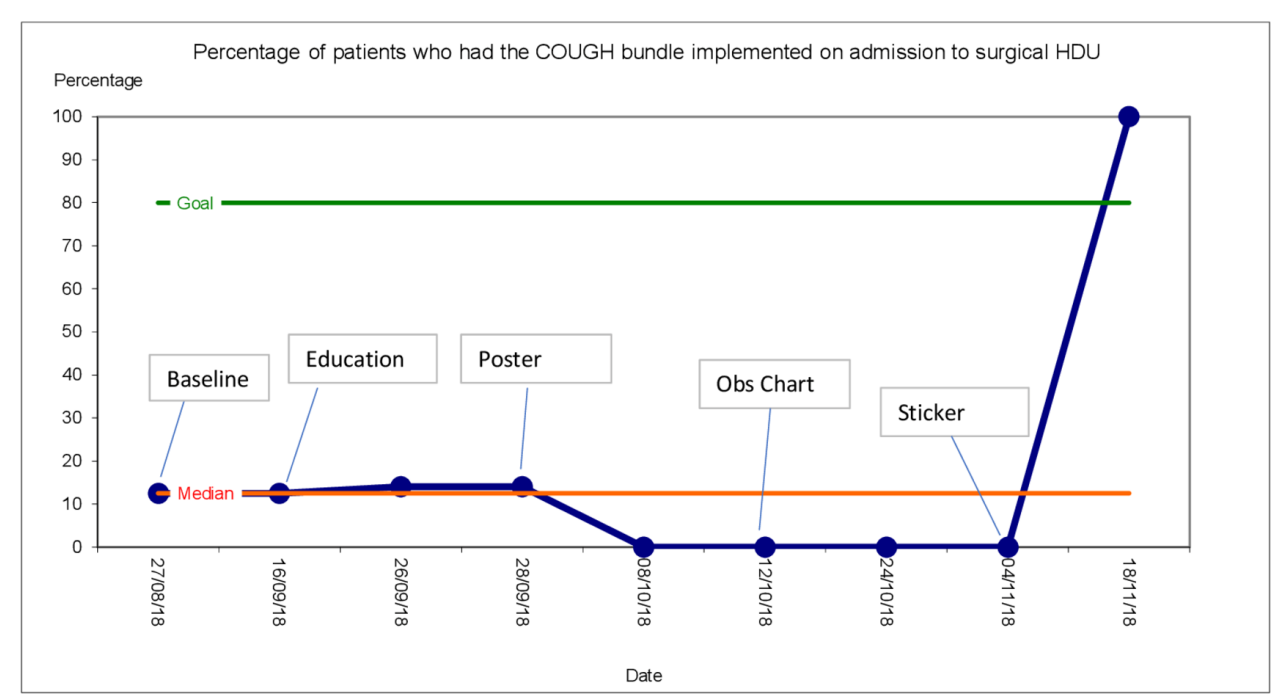

Figure 1 A graph showing the percentage of patients who had the COUGH bundle implemented on admission to surgical HDU. 
was the most successful intervention with $100 \%$ of patients receiving the COUGH bundle if indicated.

Raising awareness and educating members of the team who work in surgical HDU by sending out an email had a small success rate with the percentage of patients on the COUGH bundle increasing from $12.5 \%$ to $14 \%$. Unfortunately, the poster and moving the observation chart closer to the doctor desk were not as successful as hoped with $0 \%$ of patients receiving the COUGH bundle who should have had it. In PDSA cycle 3, only two patients had qualified for the COUGH bundle compared with other PDSA cycles which had more patients. This could have contributed to the lower percentages seen. As predicted, the sticker was the most successful intervention out of the four PDSA cycles. The sticker was placed in the surgical admission document by the ward clerk and it acted as a good reminder for staff to complete the whole of the COUGH bundle.

In order to assess whether the quality improvement project was sustainable following our interventions, data was collected 4 weeks later. The COUGH bundle was implemented in $90 \%$ of patients. This data was collected when a new set of junior doctors were rotating through the unit; therefore, this shows that the project has sustainability for the future.

Balancing measures included cost of chlorhexidine mouthwash and time taken to calculate the ARISCAT score on admission. This mouthwash according to the British National Formulary cost $£ 1.90$ for a $300 \mathrm{~mL}$ bottle. ${ }^{24}$ The average time taken to calculate the ARISCAT score for the patients included in baseline data was 3 minutes.

\section{LESSONS AND LIMITATIONS}

The project aim was to increase the use of the COUGH bundle so that patients who had a moderate or high risk of developing postoperative respiratory complications had the bundle implemented. Lessons learnt from this project included the importance of encouraging and motivating all members of the team. The most successful intervention was the sticker and this involved team work from the ward clerk, junior doctors, anaesthetists, physiotherapists and nursing staff. New junior doctors rotate throughout the unit on a 4 monthly basis; therefore, it is vital that permanent members of the team are engaged so that the project can be sustainable. Sometimes, it can be difficult for members of the team to get on board with a new project; however, we found that sending an email and including each member gave them a role to play.

Limitations of the project included that no needs assessment was performed in the initial planning of the project thus specific knowledge gaps were not addressed. Baseline data was only collected for 1 week and it would have been better if this data was collected over a longer time period. The surgical HDU has only 10 beds which meant that the number of patients included in each PDSA cycle was small. Although in the baseline data, we found that one patient who had not received the COUGH bundle, but should have, ended up developing a hospital acquired pneumonia, further data regarding postoperative respiratory complications was not collected. For future, it would have been useful to record if patients developed any postoperative respiratory complications in both those receiving the COUGH bundle and those who did not. Another limitation of the project is that one of the authors was a junior doctor within the unit when baseline data was collected which led to a higher number of patients being started on the COUGH bundle.

\section{CONCLUSION}

The COUGH bundle was designed to reduce respiratory complications in postoperative patients. Respiratory complications increase risk of morbidity and mortality but also have a financial implication as patients are likely to have an increased length of stay in hospital. This project has enabled increased awareness of the COUGH bundle and has increased the number of patients who are started on the bundle on admission to surgical HDU. The project was successful in that we met our aim and by PDSA cycle 4, $100 \%$ of patients who had an ARISCAT score of 26 or more were started on the COUGH bundle. Balancing measures included time spent calculating the ARISCAT score and the cost of the mouthwash per patient. Further data collected 1 month later showed that this project is sustainable in the future as the percentage of patients who had the COUGH bundle implemented remained high. Throughout our PDSA cycles, we have encouraged the long-standing members of the team to get involved with the project. This will allow further junior doctors to be educated when they start their placement in the unit. Furthermore, other doctors currently based in the unit are continuing to push the project forward with the development of further PDSA cycles helping to sustain it in the long term. A significant improvement has been demonstrated in this project and it may be able to be adapted to other critical care areas so that more patients can benefit.

Acknowledgements Vicki Tully, teaching lead for patient safety, Ninewells Hospital.

Contributors VL and $\mathrm{JL}$ are joint first authors - VL and $\mathrm{JL}$ contributed equally to this paper. VL and JL designed project, created PDSA cycles, collected and analysed data, drafted and edited the manuscript. SH-C reviewed manuscript and provided advice and support throughout the project.

Funding The authors have not declared a specific grant for this research from any funding agency in the public, commercial or not-for-profit sectors.

Competing interests None declared.

Patient and public involvement Patients and/or the public were not involved in the design, or conduct, or reporting, or dissemination plans of this research.

Patient consent for publication Not required.

Provenance and peer review Not commissioned; externally peer reviewed.

Data availability statement All data relevant to the study are included in the article.

Open access This is an open access article distributed in accordance with the Creative Commons Attribution Non Commercial (CC BY-NC 4.0) license, which permits others to distribute, remix, adapt, build upon this work non-commercially, and license their derivative works on different terms, provided the original work is 
properly cited, appropriate credit is given, any changes made indicated, and the use is non-commercial. See: http://creativecommons.org/licenses/by-nc/4.0/.

\section{REFERENCES}

1 Ferreyra G, Long Y, Ranieri VM. Respiratory complications after major surgery. Curr Opin Crit Care 2009;15:342-8.

2 Miskovic A, Lumb AB. Postoperative pulmonary complications. Br J Anaesth 2017:118:317-34.

3 Branson RD. The scientific basis for postoperative respiratory care. Respir Care 2013;58:1974-84.

4 Ruscic KJ, Grabitz SD, Rudolph MI, et al. Prevention of respiratory complications of the surgical patient: actionable plan for continued process improvement. Curr Opin Anaesthesiol 2017;30:399-408.

5 Cassidy MR, Rosenkranz P, McCabe K, et al. I cough: reducing postoperative pulmonary complications with a multidisciplinary patient care program. JAMA Surg 2013;148:740-5.

6 Mazo V, Sabaté S, Canet J, et al. Prospective external validation of a predictive score for postoperative pulmonary complications. Anesthesiology 2014;121:219-31.

7 Patel K, Hadian F, Ali A, et al. Postoperative pulmonary complications following major elective abdominal surgery: a cohort study. Perioper Med 2016;5:1-7.

8 Jin $\mathrm{Y}$, Xie G, Wang $\mathrm{H}$, et al. Incidence and risk factors of postoperative pulmonary complications in noncardiac Chinese patients: a multicenter observational study in university hospitals. Biomed Res Int 2015;2015:1-10.

9 Gupta S, Fernandes RJ, Rao JS, et al. Perioperative risk factors for pulmonary complications after non-cardiac surgery. $J$ Anaesthesiol Clin Pharmacol 2020;36:88-93.

10 Canet J, Gallart L, Gomar C, et al. Prediction of postoperative pulmonary complications in a population-based surgical cohort. Anesthesiology 2010;113:1338-50.

11 Fernandez-Bustamante A, Frendl G, Sprung J, et al. Postoperative pulmonary complications, early mortality, and hospital stay following Noncardiothoracic surgery. JAMA Surg 2017;152:157-66.
12 Restrepo RD, Braverman J. Current challenges in the recognition, prevention and treatment of perioperative pulmonary atelectasis. Expert Rev Respir Med 2015;9:97-107.

13 Kodra N, Shpata V, Ohri I. Risk factors for postoperative pulmonary complications after abdominal surgery. Open Access Maced J Med Sci 2016;4:259-63.

14 Kelkar KV. Post-Operative pulmonary complications after noncardiothoracic surgery. Indian J Anaesth 2015;59:599-605.

15 Tilak KM, Litake MM, Shingada KV. Study of risk, incidence and mortality associated with postoperative pulmonary complications using assess respiratory risk in surgical patients in Catalonia score. Int Surg J 2019;6:3215-22.

16 Davies OJ, Husain T, Stephens RCM. Postoperative pulmonary complications following non-cardiothoracic surgery. BJA Education 2017;17:295-300.

17 Katznelson R, Beattie WS. Perioperative smoking risk. Anesthesiology 2011;114:734-6.

18 Richardson J, Sabanathan S. Prevention of respiratory complications after abdominal surgery. Thorax 1997;52:S35-40.

19 Pöpping DM, Elia N, Marret E, et al. Protective effects of epidural analgesia on pulmonary complications after abdominal and thoracic surgery: a meta-analysis. Arch Surg 2008;143:990-9.

20 Warner DO. Preventing postoperative pulmonary complications: the role of the anesthesiologist. Anesthesiology 2000;92:1467-72.

21 Carvalho CRF, Paisani DM, Lunardi AC. Incentive spirometry in major surgeries: a systematic review. Rev Bras Fisioter 2011;15:343-50.

22 Boden I, Skinner EH, Browning L, et al. Preoperative physiotherapy for the prevention of respiratory complications after upper abdominal surgery: pragmatic, double blinded, multicentre randomised controlled trial. BMJ 2018;365:j5916.

23 Moore JA, Conway DH, Thomas N, et al. Impact of a peri-operative quality improvement programme on postoperative pulmonary complications. Anaesthesia 2017;72:317-27.

24 British National Formulary. Chlorhexidine. Available: https://bnf.nice. org.uk/medicinal-forms/chlorhexidine.html\#PHP75558 [Accessed 24 Mar 2020]. 